\title{
Data Operation with Regular
}

\section{Expression}

\author{
Roshan Jagtap \\ Dept. of Computer Science \\ DYP of College of Engineering,Pimpri \\ Pune, India
}

\author{
Rajesh Bharti \\ Dept. of Computer Science \\ DYP College of Engineering, Pimpri \\ Pune, India
}

\begin{abstract}
Regular expressions have aided as the central workhorse of practical information withdrawal for several years. The tricky of mining knowledge from huge volumes of unstructured textual info has developed increasingly significant. A vast class of element extraction activities from content that is either semi structured or completely unstructured might be inclined to by general expressions, on the estates that in frequent useful cases the suitable substances take after a basic grammatical example and this example maybe represented by a ordinary expression. The long-standing problem of manufacturing such expressions mechanically, based solely on examples of the desired behavior. To avoid this problem implement we are consuming Regex to predict a creative method of data or text handling in the flat files. Hence an enhanced files operation method in the text file can place a huge influence in the path of up gradation of the flat.
\end{abstract}

\section{Keywords}

Flat files, Synthetic files, Flat file system, Database, Regex, Text Manipulation.

\section{INTRODUCTION}

Amorphous files such as blogs, web pages, emails, and chat chats gradually form an essential source of info for data analytics [7].Regular expression is a means for stipulating string patterns briefly. Amongst all these progressive techniques we have come to be quite aware about database management system 8]. In database has searching keyword to find relevant data using regular expression matching. In database system has used to different types of data which has Oracle, SQL Server, Microsoft Access or MySQL, DB2, Paradox. Given the wide use of regular terminologies, computer procedures have been industrialized to mechanically learn them from training text samples [8]. In huge data space user key matched and find mining full data. The wide services providing by these databases are not essential and we can free ourselves from the extra work of database software Installation, meaningful a query language Generalizability is one more existing problem in regular expression. A key feature of our scheme is that the method does not necessity any clue from the user regarding the number of diverse patterns necessary foretelling the providing example [4].In dataset system extraction system has 3 issuer. They require thoughtful of the entire underlying document structure containing the data fields that the end user is not attracted in mining and their association [8]. A flat file database is database that saves data in a simple text file. Each line of the text file holds one record, with arenas separated by delimiters for commas or tabs. While it uses a simple structure, a flat file database cannot hold several tables alike a relational database can A regular expression consequently is a order of letterings that forms a search pattern, mostly for use in pattern similar with strings, In system has storage system information which has upload data stored in system.

\section{PROBLEM STATEMENT}

Knowledge a regular expression from start, consuming a incline of exact equals has been discovered. It takes also been originate to have requests in knowledge other arrangements like document type definition of XML data. Genetic algorithms modify arrangements that embody participants of a population to create a outcome that is better conferring to fitness or optimality conditions. Differentiate knowledge algorithms hip the part of info mining into stochastic-statistical methods and instruction studying approaches. Relate dissimilar field areas, or does so imperfectly, the user can intermediated by marking a structure limit around related regions. The phrase regular terminologies therefore, regexes is frequently used to mean the exact, standard written syntax for representative patterns that corresponding text need to confirm. Each variable in a regular expression is assumed to be a Metacharacter. Maintaining the Integrity of the Specifications.

\section{LITERATURE SURVEY}

Henning Fernau describes the algorithms that conclude the simple forms of unmistakable data from optimistic data regular expression. Characterized the regular linguistic learned in terms of the regular expression. Which in term in the finite automata [1].

San Jose regular expressions are used in extraction of the large amount. They has been proposed work to reduce the high quality and based on the complex operations. Proposed the ReLIE model for the more complex operation on the regular expressions. This algorithm is faster in magnitude of CRF Algorithms [2].

Alberto Bartoli et.al, learning, addressed the problem of extraction of important feature from unstructured data and problem of text slices according to the syntactical pattern. Proposed approach is based on the genetic algorithm and extracts the important feature from the data without any preprocessing. In the proposed approach main feature is that ability of finding the important feature automatically by single pattern rather than multiple patterns. Divide and conquer strategy used for the finding entity of the data and syntactic pattern, Text Patterns Using separate and Conquer Genetic Programming [3].

Josh Bongard and Hod Lipson describe the problem in the deterministic finite automata of grammatical inference and specifically assumption by the method active learning. The algorithm call estimation exploration algorithm (EEA) learning algorithm. Mentioned approach is better than previous learning approach rather than receiving training data. This algorithm EEA is most influential than the algorithms for grammatical inference, randomly-generated DFAs, on evidence driven state merging (EDSM) [4]. 
Falk Brauer et.al, described that regular expression extract the important feature from the textual data such as the invoices or product information these type of extraction follows the syntactical pattern extraction and manual pattern extraction not gives the required result related to the high precision and recall. Proposed learning method can learn effective patterns, which is easily interpreted. This approach for automatically extracting regex from the product sample information/entities derived from the enterprise database. Effectiveness is measured by feature of different granularity [5].

Alberto Bartoli explores the genetic programming for the automatic regular expression generation. Input will give by the user inn set of the task in form of text. After receiving input search text towards regular expression. Usage of text should require with regular expression for genetic genetic algorithm. Genetic programming finds the particular correct set of the regex. Obtained hopeful results in relations of two dissimilar mining tasks functional to real sphere datasets [6].

Karin Murthy raises the issue regarding the instances of with high precision and recall. Classifying false trues enclosed and changes the look to evade the false trues. The false positive is identified by the analyzing the documents manually and also find the missing instances. Focused on the finding of missing instances by analyzing user feedback. Proposed work related to the generalization of good expressions of regular expression. Presented technique for improve high precision and recall and compared over the product information [7].

Duy Duc an d An Bu et.al objective of the applying the natural language processing for the use of regular expression. Has the goal of automizing the text classification creation and utilization of regular expression. Proposed new method of regular discovering expression algorithm two text classifiers based on RED. The RED + ALIGN classifier associations RED with an alliance algorithm, and RED + SVM combines RED with a support vector machine classifier. To detect the sequential patterns classify designs could possibly be used for text sorting, though the learning itself did not accomplish classification processes [8].

Robert A Cochran et.al investigated the problem of synthesis on the crowd-sourcing. This aims to capture to find perfect solution to formalize specification. Presented approach called program boosting, combines the incorrect result by the programmers and blending this together to improve correctness. Implemented system called crowdboost this task is interesting nontrivial task like email writing. Blending of combined work outputs the boost [9].

$\mathrm{Vu}$ Le and Sumit Gulwani examines the model and view which is easy to organize the data in proper format, but sometimes makes it difficult to extract the data from it. Proposed technique Flash Extract extracts the relevant data from the unstructured data. Gives chance to user that will give the input in of the various fields to find and relate them in the hierarchical sequence and structure. By using algebra data is extracted through the synthesis algorithm by underlying specific domain [10].

\section{SCOPE OF THE PROJECT}

$\mathrm{XML}$ are widely used for storing and transporting purpose but it is not diffi- cult to understood by humans, so the proposed system will design database which easily understandable by human.

\section{GOALS AND OBJECTIVES}

Conversion of complex XML structures into Relational Database.Reducing space complexity using tree data structure.Database commits using Batch Stream Processing to fastern the process.

\section{PROPOSED SYSTEM}

Simple file or flat files are used to store the data in synthetic file database. Synthetic files are used as the database in the flat file database model. This model eliminates the complexity and take the advantage of file is the flat file, uses minimal storage space in the huge amount of data. The content of the files is filled by the user so it called as the synthetic file. The mentioned approach is that information in it is store in the single way and example can show. Expression used to denote a set of strings necessary for a scrupulous purpose. A simple way to identify a finite set of string is to list its elements or members. However, there are often more short ways to specify the preferred set of strings. In the flat files manipulation of files are used by the execution of regular expression. It is necessary to save data in the form of string and in the text file database in the specific pattern. This pattern is then used for the pattern matching gin regex Pattern matching has part in the extraction, updating, deletion and storage of data from the file.

\section{Advantages of proposed system}

1. Easy to understand and easy to implement.

2. Eliminate Complexity of data.

3. Fewer Skills set are required to hand synthetic flat

database systems best for large databases with the help of regular expression

\section{SYSTEM ARCHITECTURE}

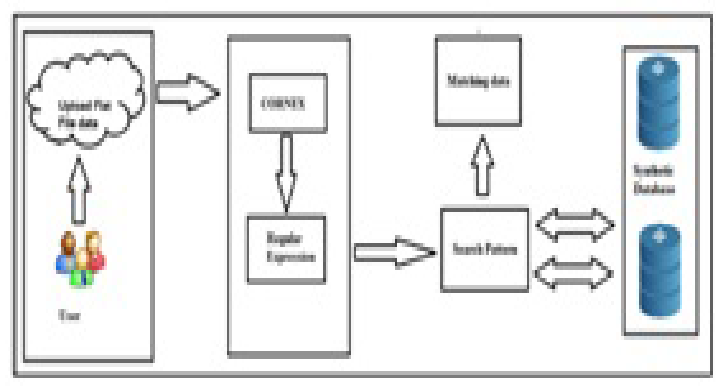

Our system is a combination of peer to peer architecture and central server. Videos will be saved on central server in form of chunks. The idea to keep video as chunks on server is to reduce load on server. User downloading file will check if there are any peers with that video. It takes into account the available parts with the other user. User will start downloading video sequentially (part-wise).

The front-end comprises of the graphical interfaces- query interface and output interface. The query interface is for the user to put his query for the data he demanded from the text file database. The query here is constrained to be grammatically correct and should only be related to the data maintained by the user in the synthetic database. The output interface is as usual responsible for displaying the results. The CONREX and SEARCH REX are the backend components, where the CONREX converts the user query into a regex pattern and then evaluates the query for its validity. The SEARCH REX comes into action only after the user query is validated and its main functionality is to search for the corresponding pattern of the CONREX generated regex pattern into the flat file database. Hence, if the match is found then the required data is displayed on the output interface otherwise an error message is reported. 


\section{ALGORITHMS \\ 1) Rabin-Karp Algorithm:}

The Rabin-Karp algorithm or Karp-Rabin algorithm is a string-searching algorithm created by Richard $M$. Karp and Michael O. Rabin (1987) that uses hashing to find any one of a set of pattern strings in a text. For text of length $\mathrm{n}$ and $\mathrm{p}$ patterns of combined length $\mathrm{m}$, its average and best case running time is $\mathrm{O}(\mathrm{n}+\mathrm{m})$ in space $\mathrm{O}(\mathrm{p})$, but its worst-case time is $\mathrm{O}(\mathrm{nm})$. In contrast, the Aho-Corasick string-matching algorithm has asymptotic worst-time complexity $\mathrm{O}(\mathrm{n}+\mathrm{m})$ in space $\mathrm{O}(\mathrm{m})$.

A practical application of the algorithm is detecting plagiarism. Given source material, the algorithm can rapidly search through a paper for instances of sentences from the source material, ignoring details such as case and punctuation. Because of the abundance of the sought strings, single-string searching algorithms are impractical.

Shifting substrings search and competing algorithms

A brute-force sub string search algorithm checks all possible positions:

This algorithm works well in many practical cases, but can exhibit relatively long running times in certain examples, such as searching for a pattern string of 10,000 "a"s followed by a single "b" in a search string of 10 million "a"s, in which case it exhibits its worst-case $\mathrm{O}(\mathrm{mn})$ time.

The Knuth-Morris-Pratt algorithm reduces this to $\mathrm{O}(\mathrm{n})$ time using pre computation to examine each text character only once; the Boyer-Moore algorithm skips forward not by 1 character, but by as many as possible for the search to succeed, effectively decreasing the number of times we iterate through the outer loop, so that the number of characters examined can be as small as $\mathrm{n} / \mathrm{m}$ in the best case.

\section{RESULT ANALYSIS}

The implemented system discussed above on the text management methodologies and developed based on illustrated GUI developed that will make user to search data stored in the database. The results retrieved based on the query given as the input to the file database. The example experiment performed on file data. It has contains number of record worker and non-worker member in company. However the department the head of the dept company who is the manager of manager. The out gets based on the designation.

$<$ id $>1<$ id $><$ name $>$ John Doe $</$ name $>$

$<$ design $>$ Head $<$ design $><$ dept $>$ BPO $<$ dept $>$

$<$ add >abs, square road, US </ add >

$<\mathrm{ph}>1234569870</ \mathrm{ph}>$

The exact query executed on the relative domain database in file results. The methodologies use on the text files. Results extracted based on the matched data in the file. Output has the whole match or partial data match and results true or false based on match.

\section{FIGURES/CAPTIONS}

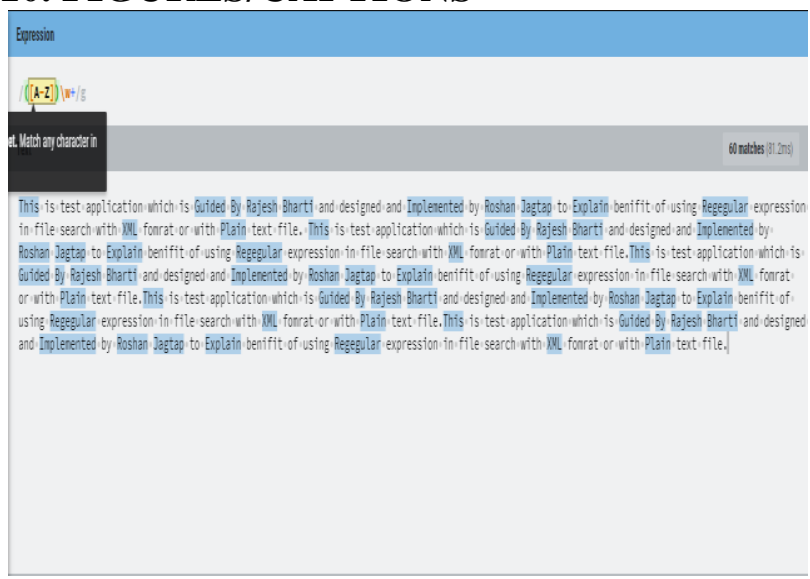

(Figure 1: Screenshot 1)

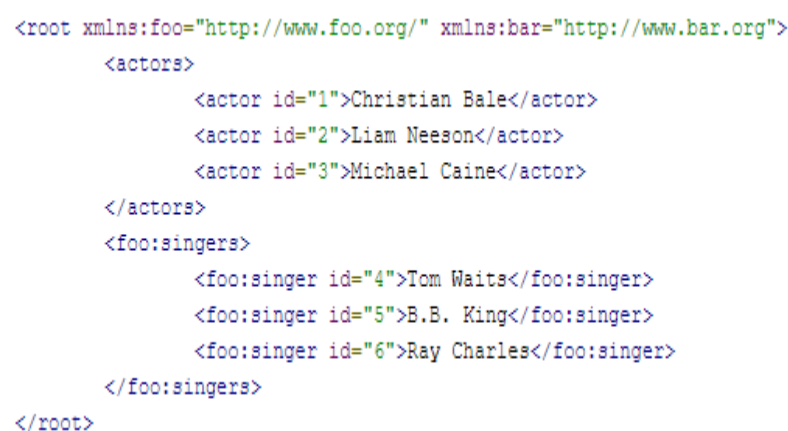

(Figure 2: Screenshot 2)

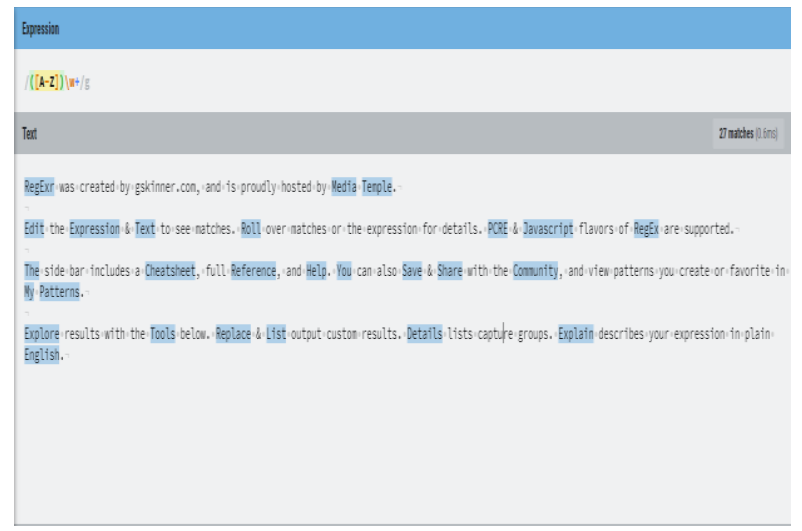

(Figure 3: Screenshot 3)

\section{CONCLUSION}

We observe and studying that a variety of frequent manuscript type such as text files, worksheets, and web pages permit handlers to be inspired here by the fundamental loaded present abilities to hoard multi-dimensional and classified information in a two dimensional outline. Presented information mining answers are domain precise and want encoding skills. We honor the difficulty of information mining in a text autonomous method and here an end handler responsive instance depend interface representation.

\section{ACKNOWLEDGMENTS}

The creators might want to thank the analysts and also for their direction. We are appreciative to the powers of Savitribai Phule University of Pune and concern individuals from CPGCON2017 gathering, sorted out by, for their steady rules and support. We are additionally appreciative to the analyst for their important 
proposals. We likewise thank the school powers for giving the required base and bolster. At long last, we might want to extend a sincere appreciation to companions and family members.

\section{REFERENCES}

[1] H. Fernau, "Algorithms for learning regular expressions from positive data," Inf. Comput., vol. 207, no. 4, pp. 521-541, 2009.

[2] Y. Li, R. Krishnamurthy, S. Raghavan, S. Vaithyanathan, and H. Jagadish, "Regular expression learning for information extraction," in Proc. Conf. Empirical Methods Natural Lang. Process., 2008,pp. 21-30.

[3] A. Bartoli, A. De Lorenzo, E. Medvet, and F. Tarlao, "Learning text patterns using separate-and-conquer genetic programming," in Proc. 18th Eur. Conf. Genetic Programm., 2015, pp. 16-27.

[4] J. Bongard, and H. Lipson. (2005). Active coevolutionary learning of deterministic finite automata. The J. Mach. Learn. Res. [Online]. 6, p. 1651-1678.

[5] F. Brauer, R. Rieger, A. Mocan, and W. M. Barczynski, "Enabling information extraction by inference of regular expressions from sample entities," in Proc. 20th ACM Int Conf. Inf. Knowl. Manage.,2011, pp. 1285-1294.

[6] A. Bartoli, G. Davanzo, A. De Lorenzo, M. Mauri, E. Medvet, and E. Sorio, "Automatic generation of regular expressions from examples with genetic programming," in Proc. 14th Annu. Conf. Companion Genetic Evol. Comput., 2012, pp. 1477-1478.

[7] K. Murthy, P. Deepak, and P. Deshpande, " Improving recall of regular expressions for information extraction," in Proc. 13th Int. Conf. Web Inf. Syst. Eng., 2012, vol. 7651, pp. 455-467.

[8] W. Contributors, "Regular expression- wikipedia, the free encyclopedia," 2015. [Online]. Available: \{https://en.wikipedia.org/wiki/Regularlexpression\}

[9] Z. contributors, "Regular expressions-user guide," 2011. [Online].

Available: \{http://www.zytrax.com/tech/web/regex.htm\}

[10] B. B. Welch, "Practical programming in tcl and tk," vol. 3rd edition Prentice Hall Professional, 1999, pp. 136-151. [Online].

Available:

\{http://www.beedub.com/book/3rd/regex.pdf $\}$

[11] J. E.F.Friedl, Mastering Regular Expressions. O'Reilly Media,Inc., 2006, vol. 3rd Edition.

[12] M. Habibi, Java Regular Expressions: Taming the java.util.regex Engine. Apress,Inc., 2004, vol. 1st Edition.

[13] Adam, "Advantages and disadvantages of flat file system," 2009. [Online]. Available: ttp://www.soopertutorials.com/technology/databases/3297advantages-disadvantages-flat-database-file-system.html \}

[14] Teach-ICT, "Limitations of flat file," 2015. [Online]. Available : $\{$ http://www.teach-ict.com/\}

[15] J. Goyvaerts and S. Levithan, Regular Expressions Cookbook. O’Reilly Media,Inc., 2012, vol. 2nd Edition.

[16] Wikipedia, "Flat file database -wikipedia, the free encyclopedia," $2015 . \quad$ [Online]. Available: \{http://en.wikipedia.org/wiki/Flat \file \database\}

[17] J. D. Cook, "Gold old regular expressions," 2010. [Online]. Available: \{http://www.johndcook.com/blog/2010/10/20/ good-old-regular-expressions/\}

[18] . S. D. Tecnologies, "Computer memory and text files," 2013.

[Online].Available: \{http://stat220.stat.auckland.ac.nz/stats220/2015/notes/200 9/ memoryandtext.ps8.pdf

[19] D. D. A. Bui and Q. Zeng-Treitler, "Learning regular expressions for clinical text classification," J. Am. Med. Informat. Assoc., vol. 21, no. 5, pp. 850-857, 2014.

[20] R. A. Cochran, L. D' Antoni, B. Livshits, D. Molnar, and M. Veanes, "Program boosting: Program synthesis via crowdsourcing," in Proc. 42nd Annu. ACM SIGPLAN-SIGACT Symp. Principles Programm. Lang., 2015, pp. 677-688.

[21] V. Le and S. Gulwani, "Flashextract: A framework for data extraction by examples," in Proc. 35th ACM SIGPLAN Conf. Programm. Lang. Des. Implementation, 2014, p. 55. 